\title{
RISK-BASED INSPECTION ON HIGHWAY ASSETS WITH CATEGORY 2 DEFECTS
}

\author{
E.O. EKPIWHRE ${ }^{1}$, K.F. TEE ${ }^{1}$, S.A. AGHAGBA ${ }^{2} \&$ K. BISHOP ${ }^{3}$ \\ ${ }^{1}$ Department of Engineering Science, University of Greenwich, UK. \\ ${ }^{2}$ Department of Highways, Ministry of Works, Delta State, Nigeria. ${ }^{3}$ Asset Management Directorate, \\ Transport for London, UK.
}

\begin{abstract}
This paper presents a study piloted on highway assets Category 2 defects. Imminent hazards on highway road networks are significantly accelerated by structural deterioration of highway infrastructures. The inspection and maintenance strategy for highway infrastructure requires continuous improvements to reduce high occurrence of defective highway assets. Combined risk-based inspection (RBI) and stochastic (STOC) techniques is considered in this investigation to give an in-depth understanding of highway asset maintenance response. Appropriate data information is extracted from Network Maintenance Management System and complementary information elicited from maintenance experts as well as recommended standards. Safety inspections piloted within the period of 5 years is evaluated using the projected RBI-STOC approach. The RBI incorporates the consequences and likelihood of the defects and the combined STOC techniques utilised defines the actual maintenance interval operated. The RBI-STOC approach proposes reclassification of highway asset defect repair intervals, appropriate maintenance task response and efficient maintenance prioritisation of highway assets in equivalence with contribution to system average mean time to repair and downtime.

Keywords: category 2 defects, probability distribution, risk-based highway assets management, riskbased inspections, safety inspection.
\end{abstract}

\section{INTRODUCTION}

A research report by Transport for London (TfL) depicts that the intervals for inspections for highway assets are principally the same notwithstanding of their strategic importance and risk of failure [1]. These inspection interval standards and strategies are not designed to cater for low risk defects on assets, thus flexibility in regimes of inspections and maintenance practice that can alter the inspection intervals, based on risk estimation is often desirable, since practical regulation is currently unavailable. Inspection regimes could be altered in recognition of a RBI approach. Inspections, surveys and assessment regimes address highway maintenance core objectives with concerns to safety, serviceability and sustainability of road network and key part of highway asset management plan development.

The importance of inspection and maintenance for system and assets of civil engineering infrastructures is of high priority and the consequences could be fatal and severe and the most importantly depreciation can be very costly [2]. A highway risk management review by Basset [3] reports that deficiencies due to defects on the road network cause approximately 5 million incidents on a yearly basis, with maintenance related defect estimated to be $95 \%$ and design defect 5\%. Lack of planned inspection and maintenance can turn new systems and assets of a road network into an environmental degenerated area and death trap. In a report by 
Adurokiya [4] on a newly constructed road indicates that fast rate, newly constructed systems could depreciate due to deprived inspection and maintenance.

This paper presents an extension of a reliability-centered maintenance strategy by Ekpiwhre and Tee [5] with the aim to support new highway infrastructures shown in Fig. 1. A risk-based inspection (RBI) and stochastic technique (RBI-STOC) for identifying suitable inspection interval for newly constructed highway assets based on similitude from RBI is projected.

The connection of quantitative risk analysis to maintenance has not been effusively studied. In addition, there is an absence of systemic, risk-based maintenance methodologies that can solve the problems facing highway agency maintenance programme. The methodology intends to enhance periodic approach to inspection and maintenance regimes, to support high reliability achievement and steady asset availability as well as to reduce downtime and hazards. The most common defect mechanism associated with highway assets can be assessed and inspection needs prioritised. The paper consists of five sections: (i) introduction, (ii) background (iii) risk-based assets management techniques, (iv) RBI-STOC case study application and finally discussion of key points and conclusion.

\section{BACKGROUND}

In July 2005, Department for Transport (DfT) [6] published a code of practice for highway maintenance management entitled the well-maintained highways. This new code replaced the first code of practice which published in 1983. The requirements set by the code are to help highway authorities addressing the problems such as the increasing growth in traffic. Inspections and surveys for highway assets, as defined by the code of practice for highway maintenance, are divided into three categories, namely safety inspection, service inspections and conditional surveys, as shown in Fig. 2.

These inspections are anticipated to identify defects with potential to cause harm, danger or serious inconvenience to road users of the network and the community environs. On site inspection is conducted to identify and assess the danger of the defect and thereafter based on extent, defects are categorised into Category 1 [CAT.1] and Category 2 [CAT.2] with appropriate response time. The risk associated with the hazards or dangers on site are identified and risk-based analysis is often developed to address the findings for this safety inspections. Risk-based methodology has been used in bridge safety serviceability and optimising the use of resources for bridge inspections [7, 8]. Safety inspections, as shown in Fig. 2, are characterised into two categories based on defect extent. CAT.2 defects are those defects requiring

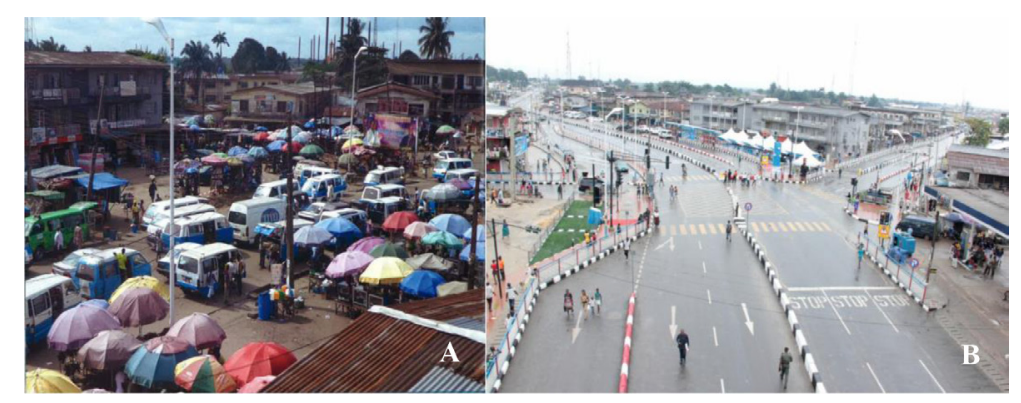

Figure 1: Drone view of Enerhen road junction network. A - Failed network, B - Newly constructed network. 


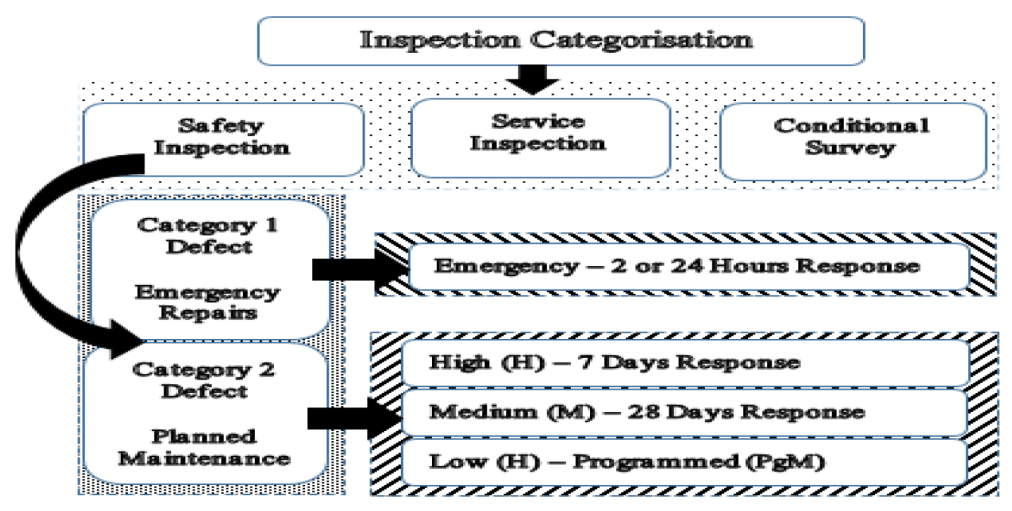

Figure 2: Highway inspections and defect categorisation.

urgent attention since they signify an immediate or imminent hazard or because there is a risk of structural deterioration within a short period. CAT.2 defects consist of all other defects deemed not to represent an immediate hazard as CAT.1 [9], and are further categorised based on priority namely high $(\mathrm{H})$, medium $(\mathrm{M})$ and low $(\mathrm{L})$.

\section{RISK-BASED ASSET MANAGEMENT}

Risk management is core requirement for achieving an optimised asset management system and it is currently becoming widely spread [10]. Highway maintenance is in need for risk analysis because of possibility of life-threatening events that can occur and lead to highway system failure arising from assets with low likelihood of occurrence [11]. Thus, there is growing need for methodologies that can support recognised and formal asset management of highway structures. Highway structures until recent times have been assured by design, assessment and maintenance in accordance with standards and regular inspections to ensure that anticipated deterioration or accidental damages have not occurred [1]. Structural failure defects are paramount consideration from any inspection and maintenance approach. Traditional methods of risk analysis on highway structures mostly consider risk implicitly and not in a manner auditable, thus leading to obfuscation of high risk or low risk assets for management purposes.

RBI and maintenance methodologies have been developed from time based maintenance through corrective maintenance [1, 12] in Fig. 3. Industries have welcomed the importance of risk-based tactics as an instrument for inspection planning and maintenance of components or assets carrying highest risk $[1,13]$. Well established, good practices and standards for the application of methodical risk analysis for systems inspection planning have been applied in bridges $[6,7,14,15]$, plant integrity in petroleum industry [12], pipelines [16, 17], mechanical systems $[18,19]$, etc.

\subsection{RBI}

RBI programme allows asset owners to prioritise and strategies their inspection activities based on the potential of defect occurring against its inherent time-based inspection regimes. Structural failure of highway infrastructure poses significant maintenance and financial burden to highway authorities in current time because of the shortage of funding leading to more 


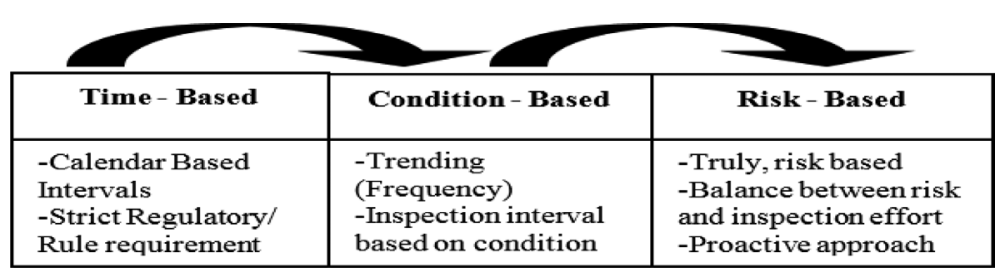

Figure 3: Inspection and maintenance planning and strategy progression.

reactive and emergency maintenance. However, failure defects and risk can be alleviated by applying RBI to maintenance as it categorises the optimal inspection interims before undesirable level of risk are stretched. RBI is beneficiary in the following ways: improves management of risk for defective systems, identifies deterioration mechanism on assets, cost reduction by eliminating unnecessary inspections, identifies effective inspection/maintenance techniques, produces an auditable system, identifies and mitigate risks over time, ensures regulatory compliance is achieved, improves asset reliability and maintainability, optimises planned downtime and ensures compliance with regulations.

\subsection{Risk assessment and analysis techniques}

Techniques used in risk assessment and analysis in literature are numerous, unique and suitable for different applications. Marhavilas \& Koulouriotis [20], points out that most risk analysis are in two categories namely deterministic (quantitative \& qualitative) and stochastic (statistics \& forecasting). The stochastic approach used is presented in Table 1.

\subsubsection{Probability distribution of repair and maintainability}

The safety and maintenance of assets and system can benefit immensely by utilisation of probability distributions from generated work site data [20, 21]. The following probability distributions with capability of dealing with the reliability of the structural health and safety of systems are presented.

3.2.1.1 Exponential distribution is one of the most used probability distribution. Systems without memory are comfortably modelled by the exponential distribution, for example, where $t>0, x>0, P(X>t+x \mid X>t)=P(X>x)$. Expression for exponentially distributed systems is shown in eqn (1) for $x \geq 0$ :

$$
f(t)=\lambda e^{(-\lambda t)}, R(t)=e^{(-\lambda t)} \text { and } \lambda(t)=\lambda
$$

3.2.1.2 Normal distribution is used for modelling the period of the lifetime of the systems. The pdf of normal distribution is given by eqn (2) where $\mu=$ average and $\sigma=$ standard deviation.

$$
f(t)=\frac{1}{\sqrt{2 \pi \sigma}} e^{-\frac{(t-\mu)^{2}}{2 \sigma^{2}}}, R(t)=\int_{t}^{\infty} f(y) \text { dy and } \lambda(t)=\frac{f(t)}{R(t)}
$$

3.2.1.3 Lognormal distribution is used to model random variables for example the repair times of systems. It relation is expressed as eqn (3). The distribution has several desirable 
Table 1: Stochastic techniques.

\begin{tabular}{ll}
\hline Categories & Analysis techniques \\
\hline Classic Statistics & Predictive Epistemic Approach (PEA) \\
Approach (CSA) & Probability Distribution - Exponential, Normal, Lognormal, Weibull \\
& Event data models - MTTF/Mean Time to Repair (MTTR) Model, Time at Risk \\
& Failure Model, Poisson Model \\
Accident Forecasting & Time-Series, Markov Chain Analysis, Grey Model, Scenario Analysis, Regression \\
Modelling (AFM) & Method, Neural Networks And Bayesian Networks \\
\hline
\end{tabular}

properties for modelling real time processes. It is positive unbound range and skewed to the right.

$$
f(t)=\frac{1}{\sqrt{2 \pi \sigma t}} e^{-\frac{(I n t-\mu)^{2}}{2 \sigma^{2}}}, t \geq 0, R(T)=\int_{t}^{\infty} f(y) d y \text { and } \lambda(t)=\frac{f(t)}{R(t)}
$$

\subsubsection{Event data models}

Stochastic behaviours of systems are better understood using MTTF/MTTR model, rate / MTTR and Time at Risk Failure model (TRF), or Poisson Model as expressed in Table 1.

3.2.2.1 Rate/MTTR model The mean time to failure is used to describe asset defects or failures discovered and repaired. It describes mean of the repair time. The parameter inputted by the user is the MTTR. The mean of its distribution is obtained by probability distributions. For exponential distribution, the MTTR $=1 / \mu$, where $\mu$ is the repair rate for an exponential distribution.

3.2.2.2 TRF model is suitable for representing only contributing components of system failures at certain periods during its duration of use. The period of unavailability associated with the model is expressed as follows, $\mathrm{Q}(\mathrm{t})=1-\mathrm{e}^{-\lambda T}$ where $\lambda$ is failure rate and is time at risk. User can specify a 'time at risk' that is different from the lifetime of the system.

\section{CASE STUDY: STOCHASTIC APPROACH ON INSPECTED ROAD NETWORKS}

Understanding of highway assets defect prioritisation involves data exploration of occurrence of three classes of CAT.2 defects developed over a period captured from real time safety inspection data and measurement of MTTR of the inspected defects. The application of RBISTOC algorithm for highway defect interval can be beneficiary for similar newly assets. Five years real time safety inspections conducted from 2010 to 2014 for highway assets in the UK are extracted from Network Maintenance Management System (NMMS).

\subsection{Risk assessment framework based on RBI-STOC approach}

The algorithm in Fig. 4 is to analyse the risk assessment for CAT.2 defects on highway assets. The RBI is conducted to identify all defects on the road network. The RBI at the outset identifies if the defect has reached a level of investigation. The investigated defect is identified as 


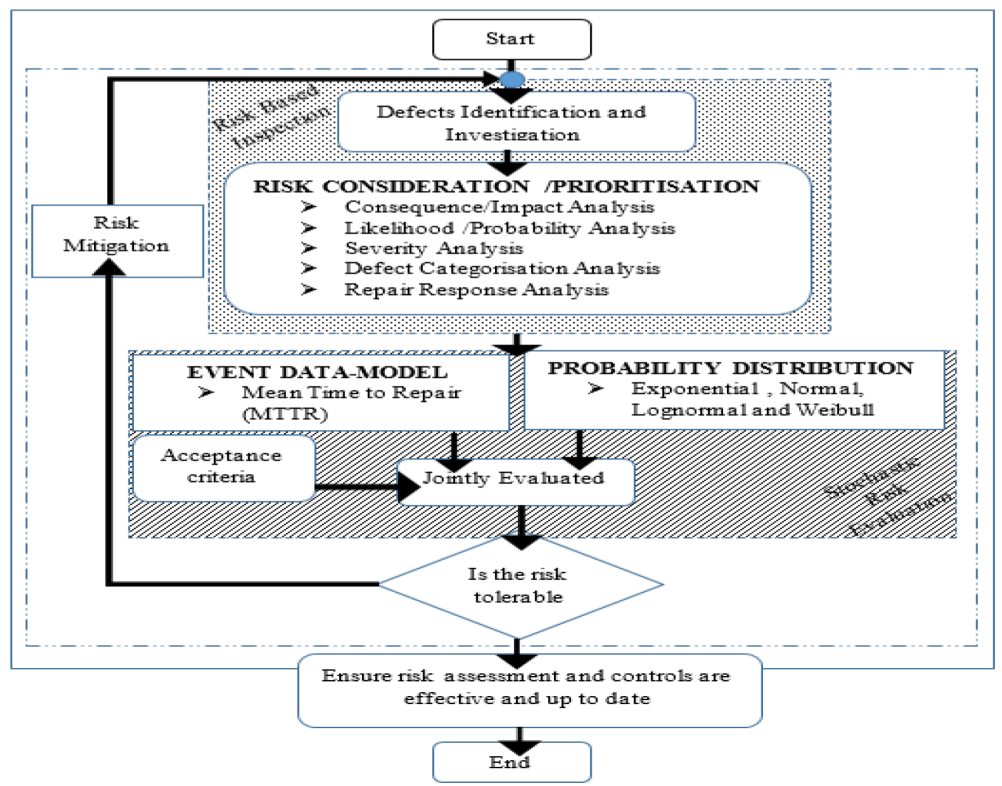

Figure 4: Flowchart of RBI-Stochastic assessment framework.

a risk if it has gotten or exceeded its investigatory level and categorised as 1, 2H, 2M or $2 \mathrm{~L}$. The RBI assesses all investigated risk-based on its significance with key focus on its impact/ consequences and probability/likelihood of the risk occurring and the probability of occurrence and in accordance with a standardised risk register. The probability of occurrence is quantified in a scale of 1 to 4 . The MTTR is obtained for various CAT.2 defects.

\subsection{Piloted risk-based safety inspection analysis}

The RBI is achieved by subjecting the occurred defects through an examination using an investigatory level benchmark to determine the risk level. The safety inspection data set is utilised to capture the key defects from the safety inspection using the investigatory standards set out in the code of conduct [6]. The inspection and repair data is essential in conducting the analysis such as defect ID, defect description, road description, defect categorisation, asset defected, cause of defect, proposed repair description, type of repair, repair date, interval meaning, etc. The average MTTR interval for CAT.2 defects is calculated and its interval mean and standard deviation for all three classes of CAT.2 defects are plotted in Fig. 5. The intervals mean represents the average repair for different defect categories. The defective period for various CAT. 2 defects from the data set is calculated and the average MTTR interval days currently in use: CAT. $2 \mathrm{H} \approx 5$ days, CAT.2M, $\approx 41$ days and CAT.2L $\approx 97$ days are illustrated in Fig. 5.

\subsubsection{The consequence/impact analysis}

The apprehended impact of the defective asset is estimated as the magnitude of the defect to the asset and the effect on the social-economic significance of the asset importance [1]. The defect score is based on various factors, such as defect size and impending level. 


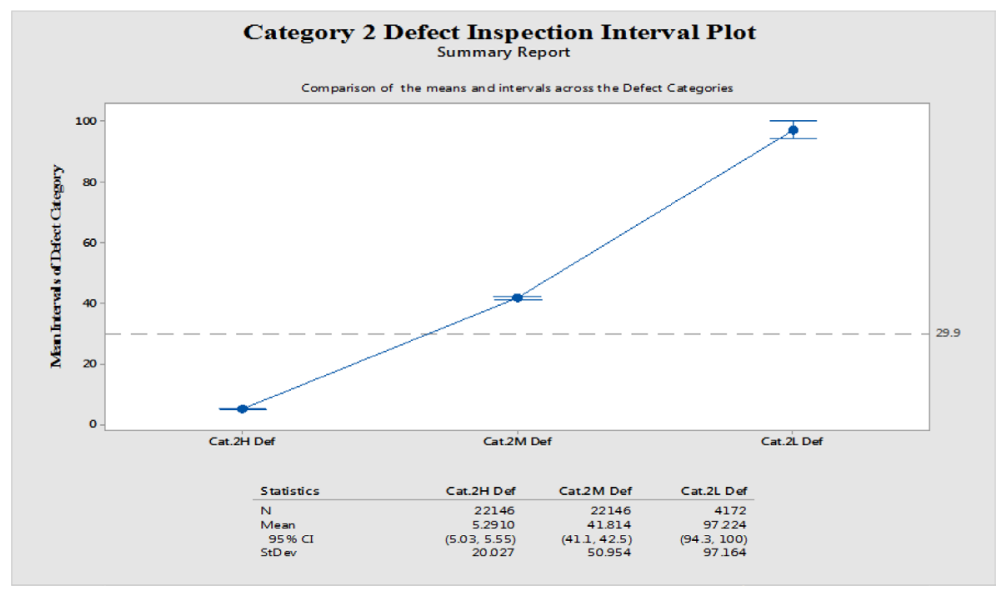

Figure 5: Means and interval relation plot piloted from RBI analysis.

\subsubsection{The likelihood/probability analysis}

The likelihood and probability of deterioration of the defect is used for scoring the defect after inspection. RBI analysis focuses on consideration of the severity of the defect and the potential failure that could arise from the defect and the assumed rate of deterioration.

\subsubsection{Defect categorisation analysis}

The defect investigated is categorised into CAT.1 or 2 based on the consequence and likelihood analysis in conjunction with the asset risk register for uniformity. The result from the risk impact calculated using eqn (4) and expressed in Table 2 is further used in prioritising maintenance response for maintenance timescale.

$$
\text { Risk level=Risk consequences } X \text { Risk probability }
$$

\subsubsection{Repair response analysis}

The defect categorisation is utilised in planning the repair response time for repairing of defective assets. The repair response is divided into three response time scale based on the risk level 1 to 9 . A risk level at 1 indicate low priority and classed lowest impact while 9 is classed as high requiring fastest response time because of its high impact and probability as shown in Table 3.

\subsection{Stochastic classical statistical approach}

The new alternative combines the proposed stochastic process (MTTR-PDF) using real time piloted safety inspection data set.

\subsubsection{Rate/ MTTR model}

The workability and predictions using the MTTR is accomplished by the integration of maintainability elements. The maintainability predictions for various defective categories are determined from the logged inspection data. At the defect category level, the MTTR 
Table 2: Risk matrix.

\begin{tabular}{|c|c|c|c|c|}
\hline \multicolumn{5}{|c|}{ Probability } \\
\hline & & Low & Medium & High \\
\hline \multirow{3}{*}{ 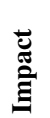 } & Low & 1 & 2 & 3 \\
\hline & Noticeable & 2 & 4 & 6 \\
\hline & High & 3 & 6 & 9 \\
\hline
\end{tabular}

Table 3: Response times for repair.

\begin{tabular}{lllll}
\hline Defect investigated & $\begin{array}{l}\text { Risk } \\
\text { level }\end{array}$ & Risk Flow & Defect priority & $\begin{array}{l}\text { Maintenance response } \\
\text { timescale }\end{array}$ \\
\hline Risk-based & $1-3$ & Low & CAT 2L & Programmed \\
inspection & $4-6$ & Medium & CAT 2M & 28 Days \\
& 9 & High & CAT 2H & 7 Days \\
\hline
\end{tabular}

maintainability elements used in computing the defective period are: (i) defect inspection/ investigated date and (ii) defect repair date. The following information from the NMMS safety inspection dataset is used to determine the assets defective period, for example date of inspection, date of work order (WO) registration, WO creation, WO instruction and date of repair. The defective period is therefore calculated using eqn (5).

$$
\text { Def }{ }^{\text {period }}=\operatorname{In}^{\text {date }}-\operatorname{Re}^{\text {date }}
$$

where Def ${ }^{\text {period }}$ is defective period, In ${ }^{\text {date }}$ is defect investigatory inspection date and $\mathrm{Re}^{\text {date }}$ is defect repaired date.

\subsubsection{Probability distribution}

Probability distributions are fundamental concept in statistics and are beneficiary both at theoretical and practical level. The mean $\mu$ and standard deviation $\sigma$ generated from MTTR interval from the sampled safety inspection data set is sampled to derive the best fit distribution trend of each defect category. The result data set is simulated via a 1,000 parametric bootstrap sampling using a $95 \%$ confidence interval level. The best fit distribution is generated using the most precise interval $\mu$ and $\sigma$, predicting the most current MTTR interval for the various defect categories. The sampled bootstrap distribution redefines the MTTR interval slightly and portrays their ideal distributions as follows: CAT. $2 \mathrm{H}: \approx 5$ days exponential distribution, CAT.2M: $\approx 65$ days lognormal distribution and CAT.2L: $\approx 97$ days exponential distribution as presented in Fig. 6.

\subsection{Joint Evaluation of RBI-STOC methodology}

The MTTR maintenance interval outcomes of the defective periods with regard to the defect categories based on the RBI and STOC analysis are presented in Table 4. The precise MTTR intervals generated via the probability distribution using a bootstrap sampling propose the recommended MTTR interval in Table 3. The MTTR and PDF provide better inspection interval regimes in consideration of the results from the MTTR-PDF outcomes. 

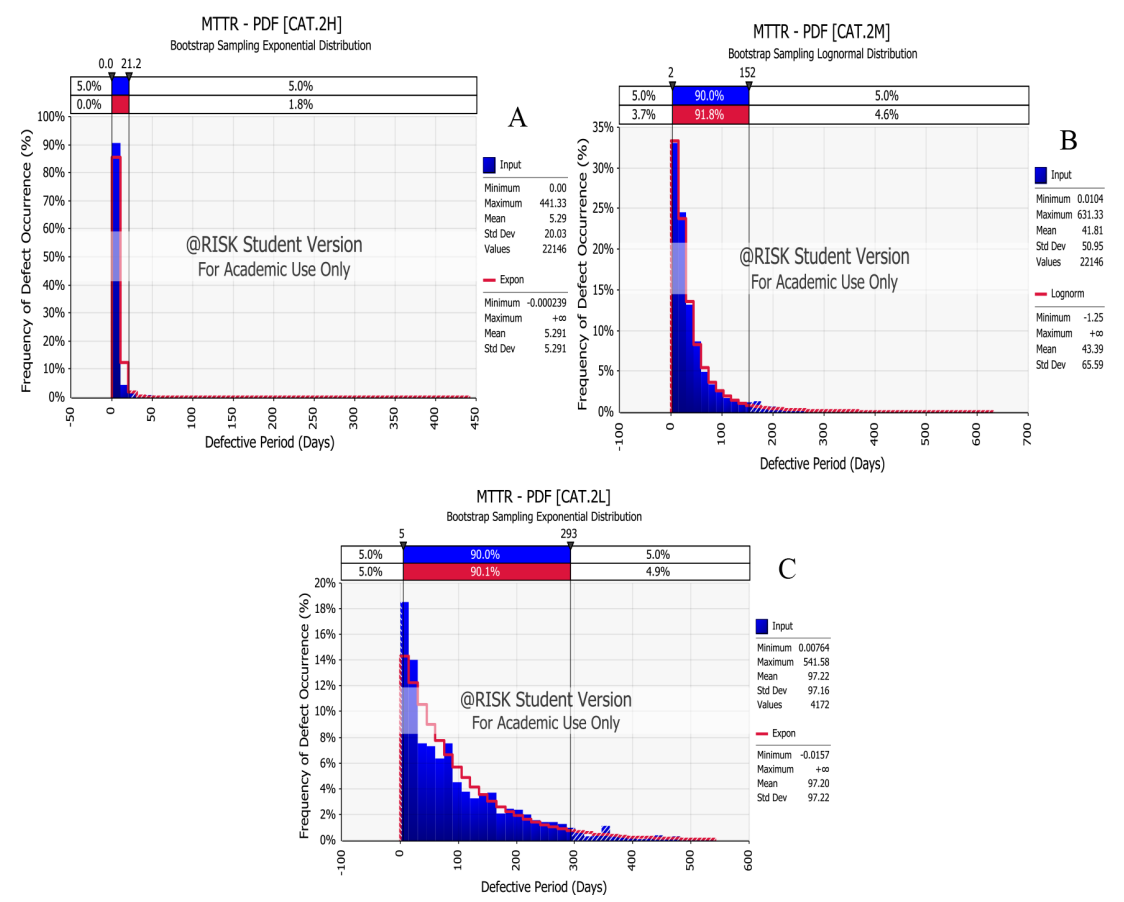

Figure 6: Parametric bootstrap sampling of PDF of CAT.2 repair interval A - CAT, 2 High, B - CAT.2 Medium, C - CAT.2 Low.

Table 4: Appending safety inspection (SI) for CAT.2 defect.

\begin{tabular}{|c|c|c|c|c|c|}
\hline \multirow[b]{2}{*}{ Priority } & \multirow{2}{*}{$\begin{array}{l}\text { Risk } \\
\text { level }\end{array}$} & \multicolumn{2}{|c|}{$\begin{array}{c}\text { Resultant stochastic } \\
\text { Interval (Days) }\end{array}$} & \multirow{2}{*}{$\begin{array}{l}\text { Present repair } \\
\text { interval (Days) }\end{array}$} & \multirow[b]{2}{*}{ Proposed RBI regime } \\
\hline & & MTTR & Best Fit PDF & & \\
\hline $2 \mathrm{~L}$ & $1-3$ & 97 & 97 - Exponential & $\operatorname{PgM}$ & Maintain SI interval \\
\hline $2 \mathrm{M}$ & $4-6$ & 42 & 65 - Lognormal & 28 & Increased SI interval \\
\hline $2 \mathrm{H}$ & 9 & 5 & 5 - Exponential & 7 & Decrease SI interval \\
\hline
\end{tabular}

\section{CONCLUSION}

The utilisation of applying a RBI approach to defects on highways assets is based on the technical foundation provided from exiting procedures of risk-based approach. RBI approach has the capability to overcome the precincts of the inherent risk practices. Inherent risk practices lack the flexibility to respond to demands by prescriptive rules and standard that is addressable by risk-based reliability methodology. RBI intertwined with reliability offers a systematic and coherent ways for dealing with risk variations and standards. Although MTTR is accurately estimated from real time field data using reliability formulas, the conception of asset operating condition and maintenance is paramount. 


\section{REFERENCES}

[1] Transport for London (Tfl), Tfl Good Practice Guide: Risk-Based Inspection of Highway Structures, 2011.

[2] Tee, K.F. \& Li, C.Q., A numerical study of maintenance strategy for concrete structures in marine environment. Proceeding of the 11th International Conference on Applications of Statistics and Probability in Civil Engineering, Zurich, Switzerland, pp. 618-625, 2011. http://dx.doi.org/10.1201/b11332-94

[3] Basset, G., Highways Risk Management Review, 2011.

[4] Adurokiya, E., 23 Months After, Multi-Million Naira Enerhen Junction Stinks. Nigeria Tribune, 2015.

[5] Ekpiwhre, E.O. \& Tee, K.F., Reliability-centered maintenance of road junction transport assets. Proceeding of the Fifteenth International Conference on Civil, Structural and Environmental Engineering Computing, eds. J. Kruis, JY. Tsompanakis \& B.H.V. Topping, Civil-Comp Press, pp. 279-289, 2015. http://dx.doi.org/10.4203/ccp.108.279

[6] Department for Transport (DfT), Well-maintained Highways: Code of Practice for Highway Maintenance Management, The Stationary Office, London, 2005.

[7] Khan, F.I., Sadiq, R. \& Haddara, M.M., Risk-based inspection \& maintenance (RBIM): multi-attribute decision-making with aggregative risk analysis. Process Safety and Environmental Protection, 82(6), pp. 398-411, 2004.

http://dx.doi.org/10.1205/psep.82.6.398.53209

[8] Washer, G., Connor, M., Nasrollahi, M. \& Provines, J., New framework for risk-based inspection of highway bridges. Journal of Bridge Engineering, 21, pp. 1-8, 2016. http://dx.doi.org/10.1061/(asce)be.1943-5592.0000818

[9] Orugbo, E.E., Alkali, B., DeSilva, A. \& Harrison, D., Reliability analysis of trunk road network maintenance: a study of category 1 defects. Advances in Manufacturing Technology XXVI, eds. T.S. Baines, B. Clegg \& D.K. Harrison., pp. 115-120, 2011.

[10] Tee, K.F. \& Khan, L.R., Risk-cost optimization and reliability analysis of underground pipelines. Proceeding of the 6th International ASRANet Conference, London, UK, 2012.

[11] Dicdican, R.Y., Haimes, Y.Y. \& Lambert, J.H., Risk-based asset management methodology for highway infrastructure systems, FHWA/VTRC 04-CR1, pp. 451-467, 2004.

[12] America Petroleum Institute (API). Risk-Based Inspection, API Recommended Practice 580, 2009.

[13] Washer, G., Connor, M., Nasrollahi, M. \& Reising, R., Verification of the framework for risk-based bridge inspection. Journal of Bridge Engineering, 21(4), 04015078, 2016. http://dx.doi.org/10.1061/(ASCE)BE.1943-5592.0000787

[14] Wintle, J.B., Kenzie, B.W., Amphlett, G.J. \& Smalley, S., Best Practice for Risk-based Inspection as a Part of Plant Integrity Management, Health \& Safety Executive (HSE) Books, 2001.

[15] Mahmoodian, M., Alani, A.M. \& Tee, K.F., Stochastic failure analysis of the gusset plates in the Mississippi river bridge. International Journal of Forensic Engineering, 1(2), pp. 153-166, 2012. http://dx.doi.org/10.1504/IJFE.2012.050415

[16] Tee, K.F., Khan, L.R., Chen, H.P. \& Alani, A.M., Reliability based life cycle cost optimization for underground pipeline networks. Tunnelling and Underground Space Technology, 43, pp. 32-40, 2014.

http://dx.doi.org/10.1016/j.tust.2014.04.007 
[17] Khan, L.R., Tee, K.F. \& Alani, A.M., Reliability-based management of underground pipeline network using genetic algorithm. Proceeding of the 11th International Probabilistic Workshop, Brno, Czech Republic, pp. 159-170, 2013.

[18] Fang, Y., Chen, J. \& Tee, K.F., Analysis of structural dynamic reliability based on the probability density evolution method. Structural Engineering and Mechanics, 45(2), pp. 201-209, 2013. http://dx.doi.org/10.12989/sem.2013.45.2.201

[19] Fang, Y., Wen, L. \& Tee, K.F., Reliability analysis of repairable k-out-n system from time response under several times stochastic shocks. Smart Structures and Systems, 14(4), pp. 559-567, 2014. http://dx.doi.org/10.12989/sss.2014.14.4.559

[20] Marhavilas, P.K. \& Koulouriotis, D.E., The Deterministic and Stochastic Risk Assessment Techniques in the Work Sites: A FTA-TRF Case Study, INTECH Open Access Publisher, 2012.

[21] Limnios, N., Fault Trees, John Wiley \& Sons, 2013. 